\title{
BIODEMOGRAFÍA EN POBLACIONES DE LA PUNA (CHAÑARCITO, SANTA ROSA DE LOS PASTOS GRANDES Y OLACAPATO) SALTA, ARGENTINA
}

\author{
BIODEMOGRAPHY OF PUNA POPULATIONS (CHAÑARCITO, SANTA ROSA \\ DE LOS PASTOS GRANDES AND OLACAPATO) SALTA, ARGENTINA
}

\author{
María Virginia Albeza*, Noemí Estela Acreche* y Graciela Beatriz Caruso*
}

\begin{abstract}
Los efectos de deriva génica, flujo génico y selección natural se estimaron a partir de parámetros demográficos que afectan la estructura genética a través de los errores de muestreo, inmigración de la fracción reproductora y oportunidad para la selección natural respectivamente. Las localidades estudiadas: Chañarcito, Santa Rosa de los Pastos Grandes y Olacapato, pertenecen al departamento Los Andes en la Provincia de Salta. Se presenta una alta probabilidad de fijación por deriva para Santa Rosa, menor pero importante para Chañarcito y por encima aunque en una pequeña fracción del límite señalado por Wright en Olacapato. La deriva génica es un factor de cambio evolutivo activo en las tres localidades. El Coeficiente de Endogamia F es elevado como consecuencia del tamaño reducido y el aislamiento de las tres localidades. El mayor Índice de Oportunidad para la Selección (I) fue estimado para Chañarcito (0,443). En Santa Rosa de los Pastos Grandes y Olacapato, I toma valores de 0,434 y 0,305 respectivamente. $\mathrm{I}_{\mathrm{f}} / \mathrm{p}_{\mathrm{s}}$ para Chañarcito es de 0,32, representando el 72,23\% del Índice total; para Santa Rosa es de 0,35, el 80,64\% y para Olacapato es de 0,20 , el $65,57 \%$, por lo que la selección se estaría ejerciendo a través de la componente de fecundidad.

Palabras claves: Biodemografía, poblaciones de la puna, tamaño efectivo poblacional, migración efectiva, coeficiente de aislamiento reproductivo, coeficiente de endogamia, oportunidad para la selección.
\end{abstract}

Genetic drift, natural selection and gene flow were analyzed by demographic parameters and were found to affect the genetic structure of populations through sampling error, immigration of the reproductive fraction and opportunity for natural selection respectively. The three small villages studied: Chañarcito, Santa Rosa de los Pastos Grandes and Olacapato, belong to the Los Andes department in the Salta Province. There exists a high probability of fixation by drift in Santa Rosa and Olacapato, lower but important in Chañarcito. Genetic drift is an active factor in the three villages. The Endogamy Coefficient $F$ is high duet to the small size and high isolation of the three small villages. The highest Opportunity Index for the Natural Selection (I) estimated was in Chañarcito (0.443). In Santa Rosa de los Pastos Grandes and Olacapato, I was 0.434 and 0.305 respectively. $I_{f} / p_{s}$ in Chañarcito is 0.32 , and $72.23 \%$ of the total Index; while the value in Santa Rosa is 0.35, 80.64\% and 0.20 (65.57\%) for Olacapato, which indicate that Selection is acting through the fecundity fraction.

Key words: Biodemography, puna's populations, effective population size, effective migration, reproductive isolation coefficient, endogamy coefficient, opportunity for selection.

La estructura por edad y sexo, la distribución espacial y temporal y los caracteres sociales, económicos y culturales son factores que poseen una relación directa con el pasado, presente y futuro biológico, social y cultural de la población. Los efectos de deriva génica, flujo génico y selección natural pueden medirse a partir de parámetros demográficos que afectan la estructura genética a través de los errores de muestreo, inmigración de la fracción reproductora y oportunidad para la selección natural respectivamente, llegando en algunos casos a provocar una microdiferenciación genética.

Los efectos de deriva génica se evalúan en función del tamaño efectivo poblacional representado por el número de individuos que han alcanzado la edad reproductora en años (Magalhaës y ArceGómez 1987a). Los errores de muestreo serán mayores en poblaciones con efectivos poblacionales reducidos, aislados, y podrán llevar a la diferenciación de la población. Los continuos desplazamientos geográficos caracterizan a nuestra especie como consecuencia de su adaptabilidad a diferentes ambientes (Valls 1985). La movilidad de los grupos de la población, es decir, por edad y sexo, ocupación, etc., es diferencial. Frecuentemente, $y$ por razones de oferta laboral entre otras, predominan migrantes de un sexo, afectando la estructura de las diferentes poblaciones.

\footnotetext{
* Consejo de Investigación de la Universidad Nacional de Salta (CIUNSa). Buenos Aires 177-4400. Salta, Argentina. mvalbeza@unsa.edu.ar
} 
Sin embargo, como no todos los individuos que entran o salen de una población se reproducen, es necesario estimar los migrantes que hacen contribuciones genéticas mediante la tasa de migración efectiva (Magalhaës y Arce-Gómez 1987a) en función de los migrantes que componen la fracción reproductora de la población. Las migraciones junto al tamaño efectivo poblacional, a través del Coeficiente de Aislamiento Reproductivo y el Coeficiente de Endogamia, permitirán evaluar la sujeción a deriva génica bajo la que se encuentra la población.

La tasa de evolución debida a selección natural se evalúa a partir del Índice de Oportunidad para la Selección, el cual incluye, como variables básicas, fecundidad y mortalidad diferencial. De este modo, la demografía genética como un nuevo enfoque de análisis de las poblaciones, permitirá, a partir de datos demográficos, conocer la estructura poblacional, comparar poblaciones y predecir cambios en el tiempo que puedan producir una diferenciación genética.

\section{Área de Estudio y Población}

Este estudio se realizó en tres localidades pertenecientes al departamento Los Andes en la provincia de Salta: Chañarcito, Santa Rosa de los Pastos Grandes y Olacapato. Chañarcito es un barrio de San Antonio de los Cobres ubicado a $165 \mathrm{~km}$ de la ciudad de Salta, con un total de 450 habitantes (136 familias) distribuidas en 137 viviendas (comunicación personal, Agente Sanitaria Plácida Mamaní); Santa Rosa de los Pastos Grandes y Olacapato se encuentran a una distancia de 45 y 40 $\mathrm{km}$ respectivamente de San Antonio de los Cobres y cuentan con una población de 114 y 149 habitantes. En Chañarcito se trabajó con una muestra $(\mathrm{N}=137)$ representativa de la población total.

El departamento Los Andes, junto al de la Poma y parte del de Rosario de Lerma, pertenecen a la región puneña. La puna se extiende por el Noroeste Argentino (NOA) entre 3.400 y 4.500 $\mathrm{msm}$. Presenta suelos inmaduros y clima frío y seco. La vegetación dominante es la estepa arbustiva con zonas de estepas herbáceas y vegas (Cabrera 1971). Entre las especies silvestres más comunes están: la tolilla (Fabiana densa), la chijua (Psila boliviensis) y la añuaga (Adesmia borridiuscula); entre las arbustivas: la rica rica (Acantholippia hastulata) y la lejía (Baccharis incarum). Por encima de los $4.000 \mathrm{msm}$ se encuentran bosques abiertos de queñoa (Polylepis tomentella), churqui (Prosopis ferox) y existen sociedades de cardones (Trichocereus pasacana, $T$. poco). Entre los cultivos se encuentran: papa (Solanum sp., Ollucus sp., Oxalis tuberosa y variedades), maíz (Zea mays) en sus tipos capia, morocho y pisingallo, haba (Vicia faba), cebolla (Allium cepa), ajo (A. sativum) y zanahoria (Daucus carota) entre otros. Las características de los sistemas de producción agrícola ganadero y minero resultan de la influencia de factores como clima, latitud, altitud, relieve y disponibilidad hídrica. $\mathrm{La}$ presencia de cuerpos permanentes de agua está condicionada a zonas en las que las precipitaciones superan los $300 \mathrm{~mm}$. Los ríos en su mayoría son de régimen pluvial. Las condiciones ambientales inducen al desarrollo de una economía con alternancia de pastoreo y agricultura en mesetas de altura y en valles y quebradas respectivamente.

La puna está caracterizada por una sociedad cuya base productiva es la unidad doméstica, dedicada a los únicos cultivos posibles adaptados a la altura y a la actividad ganadera que en conjunto conforman una economía de subsistencia. Esta sociedad ha desarrollado un complejo adaptativo en el que la transhumancia cíclica es una forma de resolver la escasez estacional de los recursos naturales, lo que resulta en un patrón de poblamiento disperso con ausencia de centros densamente poblados y la estacionalidad en la ocupación de poblados y puestos de montaña. La actividad minera tuvo gran desarrollo y fue la fuente primaria de trabajo; sin embargo, en la actualidad el sector no ofrece una salida laboral a los pobladores de la zona.

\section{Metodología}

Los datos fueron obtenidos por censo en Santa Rosa de los Pastos Grandes y Olacapato y muestreo aleatorio en Chañarcito. A partir de encuestas realizadas a las mujeres de cada vivienda, se cubrió a la totalidad de los habitantes de Santa Rosa de los Pastos Grandes y Olacapato. En el caso de Chañarcito, la muestra de la población representa el 30,45\% de los habitantes distribuidos en 18 viviendas. Las encuestas incluyen número de hogares por vivienda, número de habitantes, apellido y nombre, sexo, edad, lugar y fecha de nacimiento, estado civil, nombre y apellido del cónyu- 
ge, lugar y fecha de matrimonio, edad de fallecimiento del cónyuge (en caso de viudez), relación de parentesco entre los cónyuges, número de hijos nacidos vivos y actualmente vivos, número de hijos fallecidos, número de embarazos, nivel de instrucción y ocupación. Para cada hijo/a se consignó: nombre y apellidos del padre/madre, lugar y fecha de nacimiento; y en caso de fallecimiento, lugar, fecha y edad de fallecimiento.

A partir de esta información, se estimó: edad media de la población total, masculina y femenina y de la fracción reproductora; proporción sexual; tiempo de generación aproximado (Carnese y Caratini 1992); población reproductora $\left(\mathrm{N}_{\mathrm{r}}\right)$ considerando varones de 15 años y más y mujeres entre 15-49 años con al menos un descendiente vivo entre 0-30 años (Magalhaës y Arce-Gómez 1987a); relación niños-mujeres; tamaño poblacional efectivo según Magalhaës y Arce-Gómez (1987a) $\left(\mathrm{N}_{\mathrm{e}}=\left(2 \mathrm{~N}_{\mathrm{r}}-2\right) /\left[(\mathrm{k}-1)+\mathrm{V}_{\mathrm{k}} / \mathrm{k}^{2}\right]\right)$ y tasa de migración efectiva estimada a partir de la aproximación de Malécot (Magalhaës y Arce-Gómez 1987a), en función de los migrantes de corta y larga distancia $\left(\mathrm{m}_{\mathrm{e}}=[\mathrm{m}(\mathrm{m}+2 \mathrm{k})]^{1 / 2}\right)$.

Se incluyeron en la categoría de migrantes de largo alcance a aquellos individuos procedentes de áreas que no pertenecen a la región puneña; por el contrario, pobladores de localidades de la Puna se identificaron como migrantes de corta distancia. En función de la migración efectiva y el tamaño efectivo poblacional, se estimó el Coeficiente de Aislamiento Reproductivo $\left(\mathrm{N}_{\mathrm{e}} \mathrm{m}_{\mathrm{e}}\right)$ y el Coeficiente de Endogamia $\left(\mathrm{F}=\left(1-\mathrm{m}_{\mathrm{e}}\right)^{2} /\left[2 \mathrm{~N}_{\mathrm{e}}-\left(2 \mathrm{~N}_{\mathrm{e}}-1\right)\right.\right.$ $\left(1-\mathrm{m}_{\mathrm{e}}\right)^{2}$ ) (Magalhaës y Arce-Gómez 1987a).

Para medir la tasa de evolución por selección natural, se calculó el Índice de Oportunidad para la selección $\left(I=I_{m}+I_{f} / p_{s}\right)$ en función de la fracción de selección debida a mortalidad $\left(\mathrm{I}_{\mathrm{m}}=\mathrm{p}_{\mathrm{d}} / \mathrm{p}_{\mathrm{s}}\right)$ $\mathrm{y}$ fecundidad diferencial $\left(\mathrm{I}_{\mathrm{f}}=\mathrm{V}_{\mathrm{f}} / \mathrm{x}_{\mathrm{s}}{ }^{2}\right.$ ) (Magalhaës y Arce-Gómez 1987b).

\section{Resultados y Discusión}

No se han realizado estudios previos en las localidades estudiadas (Chañarcito, Santa Rosa de los Pastos Grandes y Olacapato). Presentan características similares en cuanto a su estructura poblacional y los efectos de factores microevolutivos a los que se hallan sujetas. La distribución por edad y sexo, en las tres localidades, refleja la estructura triangular característica y esperada para poblaciones subdesarrolladas (Magalhaës y ArceGómez 1987a). Las proporciones sexuales 90,28, 93,22 y 98,67 para Chañarcito, Santa Rosa de los Pastos Grandes y Olacapato, respectivamente, son inferiores a las registradas para la Provincia de Salta $(98,4)$ y el Departamento Los Andes $(109,0)$ (Instituto Nacional de Estadísticas y Censo [INDEC] 1994). Este indicador se ve afectado principalmente por la fuerte tendencia a emigrar en busca de nuevas ofertas laborales y en el caso de Olacapato, si bien existe una fuerte expulsión masculina, es importante el ingreso de pobladores, tanto masculinos como femeninos, por su ubicación geográfica, como área de frontera interprovincial e internacional, posibilitando constantes desplazamientos.

Son poblaciones jóvenes de acuerdo al estándar propuesto por las Naciones Unidas, ya que la población mayor de 65 años es inferior al 7\%. En Chañarcito, esta clase de edad representa el 2,92\% mientras que en Santa Rosa y Olacapato el 0,87\% y el 2,68\%. Estas estimaciones, al igual que las realizadas para otras localidades de la provincia (Acreche et al. 1994), confirman la tendencia de los países subdesarrollados a poseer bajos porcentajes en las clases de edad de 65 años y más como consecuencia, además de los continuos desplazamientos, del elevado número de hijos por mujer.

Tanto Chañarcito como Santa Rosa y Olacapato, muestran efectivos poblacionales inferiores a los registrados por Freire-Maia (Magalhaës y Arce-Gómez 1987a) para poblaciones humanas, con valores que representan el 9,42\%, 9,59\% y $10,48 \%$ respectivamente de la población total. Estos valores son muy próximos a los estimados por Acreche et al. (1994) para la localidad de San Agustín (Valle de Lerma, Salta) y Magalhaës y Arce-Gómez (1987a) para Guaraqueçaba. Los factores incidentes tales como los de desarrollo económico-social, han sido el eje de los desplazamientos hacia nuevos centros. En efecto, la explotación minera ha sido el principal factor de migración como alternativa de trabajo, provocando el movimiento de una importante fracción de la población generando cambios en la estructura demográfica.

La gran área territorial de la provincia no puede considerarse homogénea desde el punto de vista migratorio. Es necesario señalar que este fenómeno no es tampoco continuo en el tiempo, siendo fuertemente influido por causas socioeconómicas y culturales que afectan a la totalidad del territorio nacional. Salta está entre las provincias de migra- 
ción débil entre 1869 y 1960 (Rechini de Lattes y Lattes 1969). El Departamento Los Andes, según los mismos autores, se encuentra en la categoría de emigración fuerte en el período 1947-1960. Desde el punto de vista evolutivo, es éste un factor de gran importancia ya que no sólo puede incidir en el cambio de las frecuencias génicas sino que puede introducir nuevas variantes o ser causal de pérdida de algunas de ellas. Demográficamente es una variable de tanta importancia como fecundidad y mortalidad por su incidencia no sólo en el tamaño sino en las características de la población. Para el estudio de este fenómeno, tradicionalmente se han concentrado los esfuerzos en la distribución de la población en grandes centros urbanos, sin que se haya prestado demasiada importancia a los movimientos entre localidades, los que juegan un rol fundamental en la organización social y económica de los grupos establecidos en áreas como la que ocupa el presente trabajo.

Santa Rosa posee el mayor porcentaje de no migrantes. Olacapato, por su ubicación geográfica, muestra un elevado número de migrantes. En las tres localidades, el mayor porcentaje de migrantes corresponde a la categoría de corta distancia, definidos como habitantes del bioma Puna, siendo muy bajos los valores que representan a los de larga distancia (Tabla 1). Con respecto a la procedencia de los individuos que forman parte de la población reproductora $\mathrm{N}_{\mathrm{r}}$, Santa Rosa registra el menor porcentaje de migrantes (Tabla 1). Si bien, como ya se indicó, las tres localidades están aisladas geográficamente, Chañarcito y Olacapato son las que reciben un mayor número de inmigrantes como consecuencia de la pertenencia de Chañarcito al centro más poblado del departamento Los Andes (San Antonio de los Cobres) y la ubicación geográfica de Olacapato como área de frontera no sólo interprovincial sino también internacional. Asimismo, si bien son muy altos los porcentajes de desocupación, son las localidades con mejores ofertas laborales.

La mayor tasa de migración efectiva se estimó en Olacapato, mientras que la menor en Santa Rosa. En Chañarcito toma un valor intermedio (Tabla 1). Estas tasas se encuentran próximas a la estimada por Magalhaës y Arce-Gómez (1987a), 0,213, sin embargo se aleja de los valores obtenidos para nuestro país por Carnese y Caratini (1992) y Acreche et al. $(1994,1995)$ con valores de $0,97,0,43$ y 0,652 respectivamente. Wright (1938) ha sugerido la forma de medir los efectos de deriva a partir del tamaño efectivo poblacional $\mathrm{N}_{\mathrm{e}}$ y la tasa de migración efectiva $\mathrm{m}_{\mathrm{e}}$. De acuerdo a esto, valores de $\mathrm{N}_{\mathrm{e}} \mathrm{m}_{\mathrm{e}}$ inferiores a 5, implican una alta probabilidad de fijación o pérdida de genes. Por otra parte, valores entre 5-50 implican un efecto de deriva pequeño aunque no descartable.

Para las poblaciones estudiadas, se presenta en consecuencia una alta probabilidad de fijación por deriva para Santa Rosa, menor pero importante para Chañarcito y por encima, aunque en una pequeña fracción, del límite señalado por Wright en Olacapato. En esta localidad, este coeficiente se ve afectado por la tasa de migración $\mathrm{m}_{\mathrm{e}} \mathrm{y}$ el tamaño efectivo poblacional $\mathrm{N}_{\mathrm{e}}$, componentes con

Tabla 1. Chañarcito, Santa Rosa de los Pastos Grandes y Olacapato. Parámetros demográficos y genéticos. Chañarcito, Santa Rosa de los Pastos Grandes and Olacapato. Demographic and genetic parameters.

\begin{tabular}{lccc}
\hline & Chañarcito & Santa Rosa de los Pastos Grandes & Olacapato \\
\hline Población Efectiva $\left(\% \mathrm{~N}_{\mathrm{r}}\right)$ & 33,95 & 34,16 & 34,71 \\
Migración Efectiva $\left(\mathrm{m}_{\mathrm{e}}\right)$ & 0,259 & 0,231 & 0,326 \\
No Migrantes $\left(\% \mathrm{~N}_{\mathrm{r}}\right)$ & 28,95 & 43,75 & 22,22 \\
Migrantes de Corta distancia $\left(\% \mathrm{~N}_{\mathrm{r}}\right)$ & 50,00 & 37,50 & 71,11 \\
Migrantes de Larga Distancia $\left(\% \mathrm{~N}_{\mathrm{r}}\right)$ & 5,62 & 6,25 & 6,67 \\
Coeficiente de Aislamiento Reproductivo & 3,34 & 2,52 & 5,09 \\
Coeficiente de Endogamia & 0,04 & 0,02 & 0,03 \\
Índice de Oportunidad para la Selección & 0,44 & 0,43 & 0,30 \\
Componente de Mortalidad & 0,13 & 0,09 & 0,10 \\
Componente de Fecundidad & 0,28 & 0,32 & 0,19 \\
\hline
\end{tabular}


valores elevados. La deriva es entonces un factor de cambio evolutivo activo en las tres localidades (Tabla 1).

Los coeficientes de aislamiento estimados son inferiores a los que muestran las poblaciones estudiadas por Acreche et al. (1994), Carnese y Caratini (1992) y Magalhaës y Arce-Gómez (1987a), en las que los efectos de deriva son menores. Sin embargo La Alumbrera, localidad que refleja condiciones socioeconómicas similares a las localidades estudiadas, está sujeta a grandes efectos de deriva (Acreche et al. 1995). El Coeficiente de Endogamia F es elevado en las tres localidades, por lo que y de acuerdo a Cavalli-Sforza y Bodmer (1981), sería consecuencia del tamaño reducido y el aislamiento que presentan las localidades.

Chañarcito presenta el mayor coeficiente de endogamia mientras que en Santa Rosa y Olacapato los valores son muy próximos (Tabla 1). Estos valores son superiores a los estimados por Acreche et al. (1994) y Magalhaës y Arce-Gómez (1987b) con coeficientes de 0,0066 y muy próximos al de La Alumbrera $(0,010)$ (Acreche et al. 1995). Tanto los valores del Coeficiente de Aislamiento Reproductivo como el de Endogamia, medidos a través de los mismos indicadores $\left(\mathrm{N}_{\mathrm{e}} \mathrm{y} \mathrm{m}_{\mathrm{e}}\right.$ ), permiten asegurar que las localidades estudiadas se encuentran bajo la acción de deriva y flujo único. Si bien no fueron declaradas uniones consanguíneas en ninguna de las localidades, los altos Coeficientes de Endogamia revelan la posible existencia de matrimonios consanguíneos.

La tasa bruta de natalidad en el departamento Los Andes para los períodos 1980-1990 muestra un notable incremento con valores de $47,8 \%$ y $53,1 \%$ o, contraria al comportamiento para el resto de la provincia y el país. Si bien la tasa de mortalidad para la provincia disminuye en el período 1990-1992 al 6,3\% y 5,4\%o respectivamente, Los Andes también registra una disminución. No obstante, sus valores superan al del total provincial, $9,1 \%$ y $8,5 \%$ (INDEC 1994).

De acuerdo a los datos de atención primaria para la salud (1994) del hospital de San Antonio de los Cobres, que abarca los departamentos de Los Andes y La Poma (localidad de Cobres), del total de menores de 1 año (201), se registraron para 1992/93, 12 fallecimientos (5,97\%). Asimismo, es elevado el porcentaje de desnutrición para la clase de edad comprendida entre 0-5 años. En la población entre $0-2$ años, el $24 \%(\mathrm{~N}=382)$ y el $12,5 \%$
( $N=600)$ en la de 2-5 años se encuentra en diferentes canales de desnutrición. Es elevado el promedio de hijos sobrevivientes estimado para las localidades, no obstante también es alto el porcentaje de fallecidos menores de un año. Las causas de defunción en la niñez están directamente relacionadas con el reducido ingreso de los pobladores así como el alcance limitado y deficiente de los servicios más elementales de salud.

En Chañarcito el promedio de hijos sobrevivientes para mujeres y varones es de 5,32 $\left(\sigma_{\mathrm{n}-1}=2,82\right)$ y $5,41\left(\sigma_{\mathrm{n}-1}=2,53\right)$ mientras que en la fracción reproductora es de 5,27 $\left(\sigma_{\mathrm{n}-1}=2,91\right)$ $5,37\left(\sigma_{n-1}=2,60\right)$. En Santa Rosa, el promedio de hijos sobrevivientes es mayor al registrado en Chañarcito tanto para la población total como para la reproductora, siendo Olacapato la localidad que presenta el mayor promedio de hijos sobrevivientes (Tabla 2). El número medio de hijos por mujer es superior al registrado para todo el NOA en 1980 con un valor de 4,59 (Boleda 1993).

La edad promedio a la que tienen su primer hijo las mujeres es inferior en Santa Rosa con respecto a Chañarcito y Olacapato, por lo que en esta localidad una generación presenta una duración menor. Por el contrario, en Chañarcito se registró la menor edad promedio a la que tuvieron su primer hijo los varones (Tabla 2 ). $\mathrm{La}$ aproximación más directa a la selección natural consiste en medir la reproducción de los diversos genotipos en un locus y calcular en función de esto la eficacia biológica. En la práctica, como lo ha señalado Lewontin (1984), las dificultades son virtualmente insuperables. Estas dificultades son aún mayores si la especie en consideración es la nuestra, por lo menos en lo referente a adecuación genotípica.

El Índice de Oportunidad para la selección permite estimar la exposición a este factor en una población mediante la utilización de parámetros demográficos, considerando la suma de los individuos y el efecto combinado de todos los loci activos, sin apelar a las complicaciones técnicas y temporales que surgen de una aplicación clásica de la genética poblacional. En la aproximación de la genética poblacional, la adecuación biológica que se mide es la de una clase de individuos, los que poseen un determinado genotipo. En la aproximación que aquí se utiliza, genético demográfica, es una característica del conjunto de los individuos que constituyen una población. 
Tabla 2. Chañarcito, Santa Rosa de los Pastos Grandes y Olacapato. Parámetros estadísticos. Chañarcito, Santa Rosa de los Pastos Grandes and Olacapato. Statistical parameters.

\begin{tabular}{|c|c|c|c|c|c|c|}
\hline & \multicolumn{2}{|c|}{ Chañarcito } & \multicolumn{2}{|c|}{$\begin{array}{l}\text { Santa Rosa de los } \\
\text { Pastos Grandes }\end{array}$} & \multicolumn{2}{|c|}{ Olacapato } \\
\hline & Varones & Mujeres & Varones & Mujeres & Varones & Mujeres \\
\hline Población Total (N) & 65 & 72 & 55 & 59 & 74 & 75 \\
\hline Edad Media & $\begin{array}{c}16,58 \\
(15,92)\end{array}$ & $\begin{array}{c}19,54 \\
(18,87)\end{array}$ & $\begin{array}{c}17,82 \\
(16,38)\end{array}$ & $\begin{array}{c}16,24 \\
(15,27)\end{array}$ & $\begin{array}{c}17,43 \\
(17,43)\end{array}$ & $\begin{array}{c}19,70 \\
(18,60)\end{array}$ \\
\hline Edad Media Primer Hijo & $\begin{array}{l}22,50 \\
(6,19)\end{array}$ & $\begin{array}{l}18,96 \\
(4,16)\end{array}$ & $\begin{array}{l}22,93 \\
(3,97)\end{array}$ & $\begin{array}{l}17,72 \\
(1,79)\end{array}$ & $\begin{array}{l}23,18 \\
(5,53)\end{array}$ & $\begin{array}{l}19,50 \\
(3,46)\end{array}$ \\
\hline Hijos Nacidos Vivos & $\begin{array}{c}6,23 \\
(2,86)\end{array}$ & $\begin{array}{c}6,44 \\
(3,37)\end{array}$ & $\begin{array}{c}5,60 \\
(2,97)\end{array}$ & $\begin{array}{c}5,79 \\
(2,71)\end{array}$ & $\begin{array}{c}5,86 \\
(3,19)\end{array}$ & $\begin{array}{c}6,87 \\
(3,31)\end{array}$ \\
\hline Hijos Actualmente Vivos & $\begin{array}{c}5,41 \\
(2,53)\end{array}$ & $\begin{array}{c}5,32 \\
(2,82)\end{array}$ & $\begin{array}{l}4,75 \\
(2,73)\end{array}$ & $\begin{array}{c}5,26 \\
(2,97)\end{array}$ & $\begin{array}{c}5,14 \\
(2,40)\end{array}$ & $\begin{array}{c}5,79 \\
(2,50)\end{array}$ \\
\hline Población Reproductora (\% N) & 24,61 & 29,33 & 22,27 & 28,81 & 28,38 & 32,00 \\
\hline Edad Media Población Reproductora & $\begin{array}{l}37,37 \\
(9,53)\end{array}$ & $\begin{array}{c}4,95 \\
(11,75)\end{array}$ & $\begin{array}{c}34,47 \\
(12,04)\end{array}$ & $\begin{array}{c}33,70 \\
(11,25)\end{array}$ & $\begin{array}{c}39,43 \\
(14,72)\end{array}$ & $\begin{array}{c}40,31 \\
(13,42)\end{array}$ \\
\hline Promedio de Habitantes por Hogar & $\begin{array}{c}3,55 \\
(1,46)\end{array}$ & $\begin{array}{c}3,79 \\
(1,62)\end{array}$ & $\begin{array}{c}2,50 \\
(1,50)\end{array}$ & $\begin{array}{c}3,28 \\
(1,59)\end{array}$ & $\begin{array}{c}3,27 \\
(2,17)\end{array}$ & $\begin{array}{c}3,26 \\
(1,59)\end{array}$ \\
\hline
\end{tabular}

Desde la perspectiva genética, puede afirmarse con Maynard Smith (1989) que las poblaciones carecen de adecuación biológica. Este autor ha señalado también las múltiples dificultades que deben enfrentarse para medir el efecto de la selección natural mediante la adecuación biológica (efectos epistáticos, herencia poligénica con fuerte influencia ambiental, etc.).

La relación niños-mujeres (RNM) es un estimador de la fecundidad que permite establecer comparaciones entre poblaciones. Si bien este índice se refiere a un año calendario, constituye una medida de fecundidad basada en los niños sobrevivientes de las cohortes de nacidos en el quinquenio anterior a la fecha de la toma de datos y bajo los efectos de mortalidad y migración que forman parte de las limitaciones de este indicador (Camisa 1986). Es elevada la RNM estimada para las tres zonas $(928,57,1388,89$ y 1153,85 para Chañarcito, Santa Rosa y Olacapato). Estos valores son superiores a los del total de la provincia y Los Andes. Es importante el efecto de la mortalidad, evaluada a través del Índice de Oportunidad para la Selección. Del mismo modo y principalmente en
Olacapato, es notorio el impacto de las migraciones, registrándose amplios movimientos en niños de 0-4 años y mujeres en edad fértil.

La población entre 0-4 años en Chañarcito representa el $18,98 \%$ de la población, superior al registrado para el departamento Los Andes $(16,94 \%)$ (Censo Nacional de Población y Vivienda 1991); en Santa Rosa, la población que pertenece a esta clase de edad alcanza el 22,81\% del total y en Olacapato, el 20,13\%. Las mujeres en edad fértil (15-49 años) en Chañarcito representan el 38,89\% de la población femenina, en Santa Rosa, el $30,51 \%$ y en Olacapato, el 34,21\%. En el departamento Los Andes este valor alcanza el 39,91\% mientras que en la provincia, el 23,60\%. Durante el período 1947/80 en la región del NOA el porcentaje de mujeres en edad fértil, no presenta variaciones, manteniéndose en un 23\% (Boleda 1993). En la localidad de San Agustín del departamento Cerrillos, el 54\% de la población femenina se encuentra en edad fértil, mientras que en $\mathrm{La}$ Alumbrera (departamento de Andalgalá, provincia de Catamarca), este porcentaje alcanza el 35\% (Acreche et al. 1994, 1995). Esto estaría demos- 
trando, al igual que el elevado número de hijos por mujer, la elevada fecundidad que presentan las tres localidades.

El mayor Índice de Oportunidad para la selección (I) fue estimado para Chañarcito, con un valor de 0,443. En Santa Rosa de los Pastos Grandes y Olacapato, I toma valores de 0,434 y 0,305 respectivamente (Tabla 1). Las tres localidades poseen Índices inferiores a los registrados por Acreche et al. (1994) y Carnese y Caratini (1992), con valores de 0,53 y 0,66 respectivamente para San Agustín y los Tobas de Fortín Lavalle e inferiores a los de La Alumbrera, con un índice de 1,043 (Acreche et al. 1995). Este Índice estaría afectado por el elevado valor que toma la componente de mortalidad (I), en las localidades estudiadas con valores de 0,126, 0,087 y 0,100 para Chañarcito, Santa Rosa y Olacapato, respectivamente.

A pesar de la proximidad al Hospital Zonal, Chañarcito presenta el mayor Índice de Selección debido a mortalidad, lo que podría explicarse por su condición de población marginal, como ya fuera señalado, del centro más urbanizado de la zona (San Antonio de los Cobres), lo que trae aparejada la más alta tasa de desocupación de las tres poblaciones estudiadas, el mayor requerimiento de recursos provenientes de la economía de mercado y la más alta tasa de desnutrición. De acuerdo con Magalhaës y Arce-Gómez (1987b), en las poblaciones con condiciones socioeconómicas bajas, la mortalidad diferencial podría ser el principal mecanismo de selección natural.

Las localidades estudiadas son poblaciones que presentan bajas condiciones socioeconómicas, por lo que el componente de mortalidad es el esperado para una contribución tan elevada en el índice total de oportunidad para la selección. Sin embargo, la relación $\mathrm{I}_{\mathrm{f}} / \mathrm{p}_{\mathrm{s}}$ para Chañarcito es de 0,32 , representando el 72,23\% del Índice total; para Santa Rosa es de 0,35 , el $80,64 \%$ y para Olacapato es de 0,20 , el $65,57 \%$, por lo que la selección se estaría ejerciendo a través del componente de fecundidad. Es sorprendente el elevado porcentaje de desocupación detectado en las tres localidades. De los mayores de 15 años, el 47,62\%, 30,43\% y 15,62\%, para varones, y el $38,71 \%, 22,73 \%$ y $23,33 \%$, para mujeres, no realiza ninguna actividad en las localidades de Chañarcito, Santa Rosa y Olacapato. Del mismo modo, en Los Andes, también se registra la mayor tasa de analfabetismo para la población de 10 años y más con un valor de $10,6 \%$ en contrapo- sición con la del total provincial de $6,7 \%$ (INDEC 1994). En las poblaciones estudiadas, son elevadas las tasas de analfabetismo, en la población femenina de 10 años y más, es $100 \%$ mayor que el total provincial y 500\% mayor que el de la capital y en el caso de los varones, $350 \%$ mayor.

El menor valor obtenido en el índice total, estimado para Olacapato, indicaría una disminución del componente de mortalidad. Sin embargo, Santa Rosa es la localidad con menor $\mathrm{I}_{\mathrm{m}}$, por lo que se comprobaría que es la fecundidad el componente sobre el que la selección podría actuar con mayor rapidez. A pesar de los relativamente bajos valores de $I, I_{m}$ y $I_{f}$, la selección natural posee un rol importante, posiblemente característico para las poblaciones parcialmente aisladas y con condiciones socioeconómicas bajas, en las cuales el número de nacidos vivos y la mortalidad infantil alcanzan niveles muy altos, incrementados en aquellas en las que las condiciones de alfabetización son escasas o nulas como las que nos ocupan.

El estudio demográfico genético realizado en las tres localidades permite inferir que, si bien tanto Chañarcito como Santa Rosa y Olacapato presentan condiciones socioeconómicas que no satisfacen necesidades básicas de subsistencia como ya fuera mencionado, Olacapato pese a compartir esta situación es la localidad que posee un mejor nivel económico social.

Chañarcito, ubicada en el centro más urbanizado, podría ofrecer mejores condiciones de vida; la realidad y el análisis de sus datos revelan un marcado aislamiento y marginalidad, manifestado por los más altos porcentajes de no asistencia a establecimientos educacionales, de desocupación, de fecundidad y mortalidad. En el caso de Santa Rosa, si bien es una localidad aislada, sus condiciones de vida son mejores que las de Chañarcito y el análisis demográfico-genético, la ubica en una situación intermedia.

\section{Conclusiones}

Las localidades estudiadas poseen una elevada fecundidad tal y como lo demuestran el elevado número medio de hijos por progenitor y la edad de las madres al nacimiento del primer hijo. Son poblaciones jóvenes. Chañarcito y Santa Rosa de los Pastos Grandes, en función del Coeficiente de Aislamiento Reproductivo, son poblaciones que se encuentran bajo fuertes efectos de deriva génica. 
Olacapato, si bien presenta al igual que Chañarcito y Santa Rosa un reducido efectivo poblacional, es la localidad con la mayor tasa de migración efectiva lo que reduce su aislamiento y los efectos de la deriva génica. El elevado Coeficiente de Endogamia registrado en las tres localidades, es el esperado para poblaciones de tamaño reducido y aisladas, confirmando la existencia de uniones consanguíneas. Los valores estimados para las componentes de mortalidad y fecundidad del Índice de Oportunidad para la Selección demuestran que este factor estaría operando sobre la componente de fecundidad principalmente.

Agradecimientos: Agradecemos la valiosa colaboración prestada por las agentes sanitarias Plácida Mamaní y Andrea Morales de Varas, y el enfermero Hilario Morales.

\section{Referencias Citadas}

Acreche, N., y M.V., Albeza

1995 La Alumbrera: Parámetros Biodemográficos. Informe. Universidad Nacional de Salta, Salta.

Acreche, N., M.V., Albeza y G.B., Caruso

1994 San Agustín (Salta): Estructura Poblacional, Deriva y Selección. Cuadernos de Humanidades 9: 7-16.

Albeza, M.V.

1995 Deriva Génica y Estructura Demográfica en Poblaciones Andinas. Tesis Profesional (Licenciatura en Ciencias Biológicas). Facultad de Ciencias Naturales, Universidad Nacional de Salta, Salta.

Atención Primaria de la Salud.

1994 Área Operativa XXIX. Manuscrito en posesión de la autora.

Boleda, $\mathrm{M}$.

1993 La Población del Noroeste Argentino. Historia y Actualidad. Editorial Legasa/Gredes, Buenos Aires.

Boyarsky, A.

1989 Curso de Demografía. Editorial de Ciencias Sociales, La Habana.

Cabrera, A.L.

1971 Fitogeografía de la República Argentina. Boletín de la Sociedad Argentina de Botánica 15(1-2).
Camisa, Z.

1986 Introducción al Estudio de la Fecundidad. Centro Latinoamericano de Demografía, Naciones Unidas.

Carnese, F.R.

1994 Demografía Genética de la población Mapuche de Blancura Centro, Pcia. de Río Negro, Argentina. Manuscrito en posesión de la autora.

Carnese, F.

1992 Demografía genética de poblaciones aborígenes de Argentina. Revista Brasileira de Genética 15: 320-326.

Carnese, F.R., y A.Caratini

1992 Demografía genética de una población Toba de Villa Iapi (Quilmes, Prov. Buenos Aires). Etnia 36-37: 9-28.

Cavalli-Sforza, L.L., y W.F. Bodmer

1981 Genética de las Poblaciones Humanas. Editorial Omega, Barcelona.

Chávez Alvarez, E.

1989 Teoría y Política de Población. Editorial de Ciencias Sociales, La Habana.

Censo Nacional de Población y Vivienda.

1991-1993 Resultados Definitivos. Salta, Serie B No 17. INDEC. Argentina.

Instituto Nacional de Estadísticas y Censos (INDEC).

1994 Situación y Evolución Social. Síntesis Provincia de Salta (Versión Preliminar 2).

Lewontin, R.

1984 La Diversidad Humana. Biblioteca Científica American. Editorial Labor, España.

Magalhäes, J.C.M., y B., Arce-Gómez

1987a Study on a Brazilian isolate. I. Population structure and random genetic drift. Human Heredity 37: 278-284.

Magalhäes, J.C.M., y B., Arce-Gómez

1987b Study on a Brazilian isolate. II. Opportunity for selection. Human Heredity 37: 381-383.

Maynard Smith, J.

1989 Evolutionary Genetics. Oxford University Press, Oxford.

Rechini de Lattes, Z., y A. Lattes

1969 Migraciones en la Argentina. Estudio de las Migraciones Internas e Internacionales Basado en Datos Censales, 1989-1960. Editorial del Instituto Torcuato Di Tella, Buenos Aires.

Valls, A.

1985 Introducción a la Antropología. Fundamentos de la Evolución y de la Variabilidad Biológica del Hombre. Editorial Labor, España.

Wright, S.

1938 Size of population and breeding structure in relation to evolution. Science 87: 430-431. 